PROCEEDINGS OF THE

AMERICAN MATHEMATICAL SOCIETY

Volume 128, Number 6, Pages 1685-1690

S 0002-9939(99)05494-5

Article electronically published on October 27, 1999

\title{
EXTENSION THEOREMS \\ FOR THE DISTRIBUTION SOLUTIONS TO D-MODULES WITH REGULAR SINGULARITIES
}

\author{
HIROSHI KOSHIMIZU AND KIYOSHI TAKEUCHI \\ (Communicated by Lesley M. Sibner) \\ Dedicated to the Memory of Our Friend, E. Andronikof
}

\begin{abstract}
Some extension theorems for the distribution solutions to $\mathcal{D}$ modules will be given. We will use the notion of regular singularities introduced by Kashiwara-Oshima (1977).
\end{abstract}

\section{INTRODUCTION}

In this paper, we will give several extension theorems for the distribution solutions $\left(\mathrm{C}^{\infty}\right.$-solutions $)$ to $\mathcal{D}$-modules. These results are the refinements of the results for hyperfunction solutions obtained in Kashiwara-Schapira [8] and [12] (cf. also [13]), for which we make use of the characterization in [7] of the systems with regular singularities (introduced also by Kashiwara-Oshima [7]) and the ideas of [4]. One of our results is considered as a variant of the main theorem of D'Agnolo-Tonin [4].

\section{NotATiOns AND KNOWN RESUlts}

In the present paper, we essentially employ the notations and terminology of [9] and [1. Let $M$ be a real analytic manifold of dimension $n \geq 1$ and $N$ a submanifold of codimension $d \geq 1$. We take a complexification $Y \subset X$ of $N \subset M$. We denote by $\mathcal{D}_{X}$ the sheaf of holomorphic differential operators on $X$, and consider the sheaf $\mathcal{B}_{M}$ of Sato's hyperfunctions on $M$. Then we have the following results for hyperfunction solutions to coherent $\mathcal{D}_{X}$-modules.

Theorem 2.1. Let $\mathcal{M}$ be a coherent $\mathcal{D}_{X}$-module.

(i) (Kashiwara-Schapira 8]) Assume $N=\left\{x_{1}=0\right\} \subset M$ is of codimention one in $M$ and $\mathcal{M}$ is hyperbolic in the direction $+d x_{1} \in \dot{T}_{N}^{*} M$. We set $\Omega=\left\{x_{1}<\right.$ $0\} \subset M$. Then we have an isomorphism:

$$
\left.\left.R \mathcal{H o m}_{\mathcal{D}_{X}}\left(\mathcal{M}, \mathcal{B}_{M}\right)\right|_{N} \stackrel{\sim}{\longrightarrow} R \Gamma_{\Omega} R \mathcal{H} \operatorname{om}_{\mathcal{D}_{X}}\left(\mathcal{M}, \mathcal{B}_{M}\right)\right|_{N} .
$$

Received by the editors July 10, 1998.

1991 Mathematics Subject Classification. Primary 32C38.

Key words and phrases. Distribution, boundary value problem.

(C)2000 American Mathematical Society 
(ii) ([12] and [13]) We assume that the codimension $d$ of $N$ in $M$ satisfies $d \geq 2$, and consider the irreducible decomposition $\operatorname{char} \mathcal{M}=\bigcup_{j=1}^{m} V_{j}$ of the characteristic variety of $\mathcal{M}$. Suppose that each $V_{j}$ satisfies one of the following conditions:

$\left\{\right.$ (a) $V_{j}$ is non-characteristic for $Y$ and elliptic, i.e. $\dot{T}_{M}^{*} X \cap V_{j}=\emptyset$.

(b) $V_{j}$ is hyperbolic in the direction $\xi \in \dot{T}_{N}^{*} M$.

Then for every proper open convex cone $U \subset \dot{T}_{N}^{*} M$ containing $\xi$ we have the isomorphism

$$
\left.\left.\mathcal{H} \operatorname{om}_{\mathcal{D}_{X}}\left(\mathcal{M}, \mathcal{B}_{M}\right)\right|_{N} \stackrel{\sim}{\longrightarrow} \Gamma_{\Omega} \mathcal{H} \operatorname{om}_{\mathcal{D}_{X}}\left(\mathcal{M}, \mathcal{B}_{M}\right)\right|_{N}
$$

for an open tuboid $\Omega \subset M$ along $N$ satisfying the condition $U^{\circ} \cap \dot{T}_{N} M \cap$ $C_{N}(\Omega)=\emptyset$.

Let us give an example of Theorem 2.1](ii), which will explain why it cannot be stated in the derived categories (that is, in all degrees). For details, see also the proof of Theorem 4.10 of 13 .

Example 2.2 ([13]). We assume that $d=2$ and let $Q \in \mathcal{D}_{X}$ be a hyperbolic differential operator in the direction $\xi \in \dot{T}_{N}^{*} M$. We take operators $E_{j}(j=1,2)$ such that the set $V_{0}:=\bigcap_{j=1,2}\left\{\sigma\left(E_{j}\right)=0\right\} \subset T^{*} X$ is non-characteristic for $Y$ and satisfies the ellipticity condition $\dot{T}_{M}^{*} X \cap V_{0}=\emptyset$. Now we set $P_{j}=E_{j} Q+$ (lower order terms). Then for the system $\mathcal{M}=\mathcal{D}_{X} / \sum_{j=1}^{2} \mathcal{D}_{X} P_{j}$ we have by Theorem 4.10 of [13]

$$
H^{j}\left[\mu_{N} R \mathcal{H} \operatorname{om}_{\mathcal{D}_{X}}\left(\mathcal{M}, \mathcal{B}_{M}\right)\right] \simeq 0 \text { at } \quad \xi \quad \text { for } \quad j<d=2,
$$

and a local Bochner type extension theorem for hyperfunction (real analytic) solutions as in Theorem 2.1 (ii) holds. Note that $\mathcal{D}$-modules $\mathcal{M}$ obtained in this way are not hyperbolic (in the direction $\xi \in \dot{T}_{N}^{*} M$ ), nor non-characteristic for $Y$ in general. From the proof of Theorem 4.10 of [13], we cannot expect for all $j$ the vanishing of cohomologies $H^{j}\left[\mu_{N} R \mathcal{H} \operatorname{mom}_{\mathcal{D}_{X}}\left(\mathcal{M}, \mathcal{B}_{M}\right)\right]_{\xi} \simeq 0$. This is because of the elliptic factor $V_{0} \subset \operatorname{char} \mathcal{M}$.

To obtain the distribution version of the above theorem, we shall use the notion of regular singularities introduced by Kashiwara-Oshima 7] and the techniques of D'Agnolo-Tonin [4. We denote by $\mathcal{E}_{X}$ the sheaf of ring of micro-differential operators on $T^{*} X$.

Proposition 2.3 (Kashiwara-Oshima [7]). Let $V \subset \dot{T}^{*} X$ be a regular involutive submanifold and let $\mathcal{M}$ be a coherent $\mathcal{E}_{X}$-module. We take a coherent $\mathcal{E}_{X}$-module $\mathcal{S}_{V}$ which is simple along $V$. Assume that $\mathcal{M}$ has regular singularities along $V$ (in the sense of Kashiwara-Oshima []). Then we have an exact sequence

$$
\mathcal{S}_{V}^{N_{0}} \longrightarrow \mathcal{M} \longrightarrow 0
$$

of $\mathcal{E}_{X}$-modules for some $N_{0} \geq 0$. 


\section{Extension theorems For Distribution $\left(\mathrm{C}^{\infty}\right)$ SOLUTiOns}

In this section, we will give a distribution version of Theorem [2.1. Now let $\mathcal{D} b_{M}$ (resp. $\mathcal{A}_{M}$ ) be the sheaf of Schwartz's distributions (real analytic functions) on $M$ and consider the exact sequence

$$
0 \longrightarrow \mathcal{A}_{M} \longrightarrow \mathcal{D} b_{M} \underset{s p}{\longrightarrow} \dot{\pi}_{*} \mathcal{C}_{M}^{f} \longrightarrow 0
$$

where $\mathcal{C}_{M}^{f}$ is the sheaf of tempered microfunctions introduced by Bony [2] (see also Andronikof [1] for a functorial construction) and $\dot{\pi}: \dot{T}_{M}^{*} X \longrightarrow M$ is the projection. In the first part of the next theorem, we consider the systems introduced by D'Agnolo-Tonin 4 .

Theorem 3.1. Let $\mathcal{M}$ be a coherent $\mathcal{D}_{X}$-module.

(i) We consider the same situation as in Theorem 2.1 (i). Assume moreover:

(a) $V_{\mathbf{R}}=$ char $\mathcal{M} \cap \dot{T}_{M}^{*} X$ is a smooth regular involutive submanifold of $\dot{T}_{M}^{*} X$ such that $V=$ char $\mathcal{M}$ is a complexification of $V_{\mathbf{R}}$ in an open neighborhood of $\dot{T}_{M}^{*} X$ in $T^{*} X$.

(b) As a coherent $\mathcal{E}_{X}$-module, $\mathcal{E}_{X} \otimes_{\pi_{X}^{-1} \mathcal{D}_{X}} \pi_{X}^{-1} \mathcal{M}\left(\pi_{X}: T^{*} X \longrightarrow X\right)$ has regular singularities along $V=\operatorname{char} \mathcal{M}$ on $V_{\mathbf{R}}$.

Then we have an isomorphism:

$$
\left.\left.R \mathcal{H} \operatorname{om}_{\mathcal{D}_{X}}\left(\mathcal{M}, \mathcal{D} b_{M}\right)\right|_{N} \stackrel{\sim}{\longrightarrow} R \Gamma_{\Omega} R \mathcal{H} \operatorname{Hom}_{\mathcal{D}_{X}}\left(\mathcal{M}, \mathcal{D} b_{M}\right)\right|_{N} .
$$

(ii) We consider the same situation as in Theorem 2.1 (ii) and assume that the conditions (a) and (b) in (i) above are satisfied. Then for every proper open convex cone $U \subset \dot{T}_{N}^{*} M$ containing $\xi \in \dot{T}_{N}^{*} M$ we have the isomorphism

$$
\left.\left.\mathcal{H} \operatorname{om}_{\mathcal{D}_{X}}\left(\mathcal{M}, \mathcal{D} b_{M}\right)\right|_{N} \stackrel{\sim}{\longrightarrow} \Gamma_{\Omega} \mathcal{H} \operatorname{Hom}_{\mathcal{D}_{X}}\left(\mathcal{M}, \mathcal{D} b_{M}\right)\right|_{N}
$$

for an open tuboid $\Omega \subset M$ along $N$ satisfying the condition $U^{\circ} \cap \dot{T}_{N} M \cap$ $C_{N}(\Omega)=\emptyset$.

Proof. To explain the idea of the proof, first we restrict ourselves in proving formula (3.2) in the "0-th cohomology"

$$
\left.\left.\mathcal{H}_{\mathrm{om}_{\mathcal{D}_{X}}}\left(\mathcal{M}, \mathcal{D} b_{M}\right)\right|_{N} \stackrel{\sim}{\sim} \Gamma_{\Omega} \mathcal{H}_{\mathrm{om}_{\mathcal{D}_{X}}}\left(\mathcal{M}, \mathcal{D} b_{M}\right)\right|_{N}
$$

(i) We take a section $\left.u \in \Gamma_{\Omega} \mathcal{H}_{\mathrm{om}} \mathrm{D}_{X}\left(\mathcal{M}, \mathcal{D} b_{M}\right)\right|_{N}$ and consider the extension $\left.\tilde{u} \in \mathcal{H} \operatorname{om}_{\mathcal{D}_{X}}\left(\mathcal{M}, \mathcal{B}_{M}\right)\right|_{N}$ of $u$ as a hyperfunction solution, which is possible thanks to Theorem 2.1 (i). Now we have to verify that the extension $\tilde{u}$ is again a distribution. For this purpose, we regard $\tilde{u}$ as a section of the sheaf $\mathcal{C}_{M}$ of microfunctions on $\dot{T}_{M}^{*} X$ which is contained in $\mathcal{C}_{M}^{f}$ on the half side $\dot{\pi}^{-1}(\Omega) \subset \dot{T}_{M}^{*} X$. It follows from the assumptions (a), (b) and Proposition 2.3 that the $\mathcal{E}_{X}$-module $\tilde{\mathcal{M}}=\mathcal{E}_{X} \otimes_{\pi_{X}{ }^{-1} \mathcal{D}_{X}} \pi_{X}{ }^{-1} \mathcal{M}$ can be locally transformed to a quotient of a direct sum of the de Rham system by a real contact transformation $\Phi: U_{1} \simeq U_{2}$ between two open subsets $U_{1}, U_{2} \subset \dot{T}_{M}^{*} X$. We denote by $\mathcal{S}$ this de Rham system. Then we have an exact sequence $\mathcal{S}^{N_{0}} \longrightarrow \Phi_{*} \tilde{\mathcal{M}} \longrightarrow 0$ of $\mathcal{E}_{X}$-module for some $N_{0} \geq 0$ on $U_{2}$ and the commutative diagram

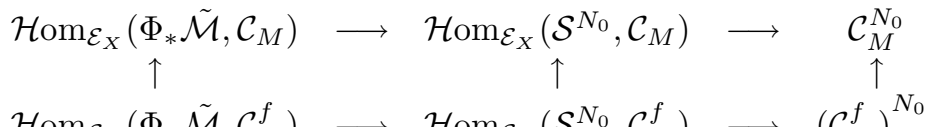

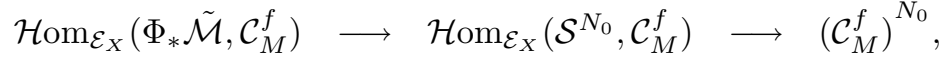


in which all arrows are injective. Since the section

$$
\Phi_{*} \tilde{u} \in \Gamma\left(U_{2} ; \mathcal{H}^{\circ} m_{\mathcal{E}_{X}}\left(\mathcal{S}^{N_{0}}, \mathcal{C}_{M}\right)\right)
$$

is contained in $\left(\mathcal{C}_{M}^{f}\right)^{N_{0}}$ on the half side $\Phi\left(\dot{\pi}^{-1}(\Omega) \cap U_{1}\right) \subset U_{2}$ of $U_{2}$ and "constant" along real bicharacteristic strips of $\mathcal{S}$ which are transversal to $\Phi\left(\dot{\pi}^{-1}(N) \cap\right.$ $\left.U_{1}\right), \Phi_{*} \tilde{u}$ is contained in $\left(\mathcal{C}_{M}^{f}\right)^{N_{0}}$ also on $\Phi\left(U_{1}\right)=U_{2}$. Therefore we get $\Phi_{*} \tilde{u} \in$ $\Gamma\left(U_{2} ; \mathcal{H}^{\circ} \mathrm{m}_{\mathcal{E}_{X}}\left(\Phi_{*} \tilde{\mathcal{M}}, \mathcal{C}_{M}^{f}\right)\right)$, and the invariance by quantized contact transformations of the sheaf $\mathcal{C}_{M}^{f}$ proved by Bony 2] (see also Andronikof [1]) ensures that $\tilde{u} \in \Gamma\left(U_{1} ; \mathcal{H}^{\circ} m_{\mathcal{D}_{X}}\left(\mathcal{M}, \mathcal{C}_{M}^{f}\right)\right)$. This implies $\left.\tilde{u} \in \mathcal{H}^{\prime} \mathrm{m}_{\mathcal{D}_{X}}\left(\mathcal{M}, \mathcal{D} b_{M}\right)\right|_{N}$ and completes the proof of the 0 -th cohomology part of (i).

The part (ii) is also proved by extending $\left.u \in \Gamma_{\Omega} \mathcal{H}_{\mathrm{om}} \overline{\mathcal{D}}_{X}\left(\mathcal{M}, \mathcal{D} b_{M}\right)\right|_{N}$ as a hyperfunction solution (by Theorem 2.1 (ii)) and using the same microlocal observation as above.

Finally, we will prove formula (3.2) completely; that is, in the derived category. Since the system $\mathcal{M}$ in consideration is now hyperbolic, we may apply Corollary 6.4.4. of 9] to get the isomorphism

$$
\left.\left.R \mathcal{H}_{\mathrm{om}_{\mathcal{X}}}\left(\mathcal{M}, \mathcal{A}_{M}\right)\right|_{N} \stackrel{\sim}{\longrightarrow} R \Gamma_{\Omega} R \mathcal{H}_{\mathrm{om}_{\mathcal{D}_{X}}}\left(\mathcal{M}, \mathcal{A}_{M}\right)\right|_{N}
$$

Hence by considering Sato's distinguished triangle, it remains to show

$$
R \Gamma\left(U ; R \dot{\pi}_{*} R \mathcal{H} \operatorname{lom}_{\mathcal{D}_{X}}\left(\mathcal{M}, \mathcal{C}_{M}^{f}\right)\right) \stackrel{\sim}{\longrightarrow} R \Gamma\left(U \cap \Omega ; R \dot{\pi}_{*} R \mathcal{H} \operatorname{om}_{\mathcal{D}_{X}}\left(\mathcal{M}, \mathcal{C}_{M}^{f}\right)\right)
$$

for an open neighborhood $U$ of $N$. But it follows from the Mayer-Vietoris argument that the proof of the isomorphism (3.7) can be reduced to the one

$$
R \Gamma\left(U_{1} ; R \mathcal{H}_{\mathrm{om}_{\mathcal{D}_{X}}}\left(\mathcal{M}, \mathcal{C}_{M}^{f}\right)\right) \stackrel{\sim}{\longrightarrow} R \Gamma\left(U_{1} \cap \dot{\pi}^{-1}(\Omega) ; R \mathcal{H}_{\mathrm{om}_{\mathcal{D}_{X}}}\left(\mathcal{M}, \mathcal{C}_{M}^{f}\right)\right)
$$

for a sufficiently small open subset $U_{1} \subset \dot{T}_{M}^{*} X$, which is equivalent to

$$
R \Gamma\left(U_{2} ; R \mathcal{H} \operatorname{om}_{\mathcal{E}_{X}}\left(\Phi_{*} \tilde{\mathcal{M}}, \mathcal{C}_{M}^{f}\right)\right) \stackrel{\sim}{\longrightarrow} R \Gamma\left(\Phi\left(U_{1} \cap \dot{\pi}^{-1}(\Omega)\right) ; R \mathcal{H} \operatorname{om}_{\mathcal{E}_{X}}\left(\Phi_{*} \tilde{\mathcal{M}}, \mathcal{C}_{M}^{f}\right)\right)
$$

through the real contact transformation $\Phi: U_{1} \simeq U_{2}$ in the proof above. If we consider a resolution of the $\mathcal{E}_{X}$-module $\Phi_{*} \tilde{\mathcal{M}}$ (by the same de Rham system $\mathcal{S}$ )

$$
\longrightarrow \mathcal{S}^{N_{2}} \longrightarrow \mathcal{S}^{N_{1}} \longrightarrow \mathcal{S}^{N_{0}} \longrightarrow \Phi_{*} \tilde{\mathcal{M}} \longrightarrow 0
$$

by the repeated use of Proposition 2.3, we can prove this last isomorphism (3.9) by a standard argument and the formula

$$
R \Gamma\left(U_{2} ; R \mathcal{H}^{\circ} \dot{\mathcal{E}}_{X}\left(\mathcal{S}, \mathcal{C}_{M}^{f}\right)\right) \stackrel{\sim}{\longrightarrow} R \Gamma\left(\Phi\left(U_{1} \cap \dot{\pi}^{-1}(\Omega)\right) ; R \mathcal{H} \operatorname{om}_{\mathcal{E}_{X}}\left(\mathcal{S}, \mathcal{C}_{M}^{f}\right)\right)
$$

for the de Rham system $\mathcal{S}$. Note that the same argument was also used in D'AgnoloTonin 4 to solve the Cauchy problem for distributions.

Remark 3.2. The same situation as in Theorem 3.1 (i) was first considered by D'Agnolo-Tonin 4]. In fact, we have an isomorphism

$$
\left.\left.R \mathcal{H}_{\mathrm{om}_{\mathcal{D}_{X}}}\left(\mathcal{M}, \mathcal{D} b_{M}\right)\right|_{N} \stackrel{\sim}{\longrightarrow} R \mathcal{H}_{\mathrm{om}_{\mathcal{D}_{X}}}\left(\mathcal{M}, \operatorname{Thom}\left(\mathbb{C}_{\Omega}, \mathcal{D} b_{M}\right)\right)\right|_{N}
$$


as an immediate cosequence of their main theorem, where $T h o m\left(*, \mathcal{D} b_{M}\right)$ is the functor $T H$ of Kashiwara [ $\left[6\right.$ and $T h o m\left(\mathbb{C}_{\Omega}, \mathcal{D} b_{M}\right)$ denotes the subsheaf of $\Gamma_{\Omega} \mathcal{D} b_{M}$ consisting of sections which are extendible through $N$ as distributions. Our Theorem 3.1 (i) removes this growth condition on the function space.

Remark 3.3. Theorem 3.1(i) can be restated in the following way. Let $j: T^{*} M \longrightarrow$ $T^{*}\left(T_{M}^{*} X\right) \simeq T_{\left(T_{M}^{*} X\right)}\left(T^{*} X\right)$ be the injection induced by the projection $T_{M}^{*} X \longrightarrow M$, where we used the Hamiltonian isomorphism $-H: T^{*}\left(T_{M}^{*} X\right) \simeq T_{\left(T_{M}^{*} X\right)}\left(T^{*} X\right)$. Then one of the main results of Kashiwara-Schapira [8] was the following estimation of the micro-support of the hyperfunction solution complexes:

$$
\operatorname{SS}\left[R \mathcal{H}_{\mathcal{D}_{X}}\left(\mathcal{M}, \mathcal{B}_{M}\right)\right] \subset j^{-1} C_{\left(T_{M}^{*} X\right)}(\operatorname{char} \mathcal{M}),
$$

which is valid for arbitrary coherent $\mathcal{D}_{X}$-module $\mathcal{M}$. Our Theorem 3.1 (i) is the distribution version of this result. That is, if we take a coherent $\mathcal{D}_{X}$-module $\mathcal{M}$ satisfying the conditions (a) and (b) of Theorem 3.1 (i), then we have

$$
\mathrm{SS}\left[R \mathcal{H}_{\mathrm{om}_{\mathcal{D}_{X}}}\left(\mathcal{M}, \mathcal{D} b_{M}\right)\right] \subset j^{-1} C_{\left(T_{M}^{*} X\right)}(\operatorname{char} \mathcal{M}) .
$$

From the proof of Theorem 3.1 we also get the following Hartogs type theorem for distribution solutions, which is a refinement of the division theorem of Kashiwara [5] and Kawai [10.

Theorem 3.4. Assume that the codimension $d$ of $N$ in $M$ satisfies $d \geq 2$. Let $\mathcal{M}$ be a coherent $\mathcal{D}_{X}$-module for which $Y$ is non-characteristic. We also assume that $\mathcal{M}$ satisfies the conditions (a) and (b) of Theorem 3.1 (i). Then we have an isomorphism

$$
\left.\left.\mathcal{H o m}_{\mathcal{D}_{X}}\left(\mathcal{M}, \mathcal{D} b_{M}\right)\right|_{N} \stackrel{\sim}{\longrightarrow} \Gamma_{M-N} \mathcal{H o m}_{\mathcal{D}_{X}}\left(\mathcal{M}, \mathcal{D} b_{M}\right)\right|_{N} .
$$

Remark 3.5. We can replace the sheaf $\mathcal{D} b_{M}$ in Theorems 3.1 and 3.4 by the sheaf $\mathcal{C}_{M}^{\infty}$ of $\mathrm{C}^{\infty}$-functions. Since Colin [3] recently proved the invariance of the sheaf of differentiable microfunctions of Bony [2] through real quantized contact transformations, the proof of Theorem 3.1 applies also to the case of $\mathrm{C}^{\infty}$-functions.

\section{REFERENCES}

[1] E. Andronikof, Microlocalisation tempérée, Mém. Soc. Math. France, 57 (1994). MR 95e:58168

[2] J.M. Bony, Propagation des singularités microlocale analytique des distributions, Astérisque, 34-35 (1976), 43-91. MR 58:23199

[3] V. Colin, Thése at Paris VI, and a note to appear, (1997).

[4] A. D'Agnolo and F. Tonin, Cauchy problem for hyperbolic $\mathcal{D}$-modules with regular singularities, Pacific Journal of Mathematics, 184 (1998), 1-22. CMP 98:13

[5] M. Kashiwara, Algebraic study of systems of linear differential equations (Master Thesis, Tokyo Univ., 1970), Mém. Soc. Math. France, 63 (1995). MR 97f:32012

[6] M. Kashiwara, The Riemann-Hilbert problem for holonomic systems, Publ. Res. Inst. Math. Sci., 20 (1984), 319-365. MR 86j:58142

[7] M. Kashiwara and T. Oshima, Systems of differential equations with regular singularities and their boundary value problems, Ann. Math., 106 (1977), 145-200. MR 58:2914

[8] M. Kashiwara and P. Schapira, Micro-hyperbolic systems, Acta Math., 142 (1979), 1-55. MR 80b:58060

[9] M. Kashiwara and P. Schapira, Sheaves on manifolds, Grundlehlen der Math. Wiss., 292, Springer-Verlag (1990). MR 92a:58132

[10] T. Kawai, Extension of solutions of systems of linear differential equations, Publ. Res. Inst. Math. Sci., 12 (1976-77), 215-227. MR 54:3767

[11] M. Sato, T. Kawai and M. Kashiwara, Hyperfunctions and pseudodifferential equations, L.N. in Math., Springer-Verlag, 287 (1973), 265-529. 
[12] K. Takeuchi, Edge-of-the-wedge type theorems for hyperfunction solutions, Duke Math. J., 89 (1997), 109-132. MR 98h:32015

[13] K. Takeuchi, Microlocal inverse image and bimicrolocalization, Publ. Res. Inst. Math. Sci., 34 (1998), 135-153. MR 99f:32018

Department of Mathematical Sciences, University of Tokyo, 3-8-1, Komaba, Meguro$\mathrm{KU}, 153$, JAPAN

Department of Mathematics, Hiroshima University, 1-3-1, Kagamiyama, Higashi-hiroSHIMA, Hiroshima, 739-8526, JaPAN

E-mail address: takeuchi@top2.math.sci.hiroshima-u.ac.jp 